\title{
GEODESICS IN THE SPACE OF KÄHLER METRICS
}

\author{
LÁSZLÓ LEMPERT ${ }^{1}$ AND LIZ VIVAS
}

\begin{abstract}
Let $(X, \omega)$ be a compact Kähler manifold. As discovered in the late 1980s by Mabuchi, the set $\mathcal{H}_{0}$ of Kähler forms cohomologous to $\omega$ has the natural structure of an infinite dimensional Riemannian manifold. We address the question whether any two points in $\mathcal{H}_{0}$ can be connected by a smooth geodesic, and show that the answer, in general, is "no".
\end{abstract}

\section{Introduction.}

Let $X$ be a connected, compact, complex manifold and $\omega_{0}$ a smooth Kähler form on it. It was discovered by Mabuchi, and rediscovered by Semmes and Donaldson, that the set $\mathcal{H}_{0}$ of smooth Kähler forms cohomologous to $\omega_{0}$, and the set $\mathcal{H}$ of smooth, strongly $\omega_{0}$-plurisubharmonic functions on $X$ carry natural infinite dimensional Riemannian manifold structures, see [M,S,D1].- A function $u: X \rightarrow \mathbb{R}$ is (strongly) $\omega_{0}$-plurisubharmonic if $\omega_{0}+i \partial \bar{\partial} u \geq 0$ (resp. $>0$ ). - Mabuchi shows that in fact $\mathcal{H}$ is isometric to the Riemannian product $\mathcal{H}_{0} \times \mathbb{R}$, and both he and Donaldson point out that understanding geodesics in these spaces is important for the study of special Kähler metrics. Donaldson then raises the obvious question whether any pair of points in $\mathcal{H}\left(\right.$ or $\left.\mathcal{H}_{0}\right)$ can be connected by a smooth geodesic. In this paper we give a negative answer:

Theorem 1.1. Suppose $\left(X, \omega_{0}\right)$ is a positive dimensional connected compact Kähler manifold and $h: X \rightarrow X$ is a holomorphic isometry with an isolated fixed point such that $h^{2}=i d_{X}$. Then there is a Kähler form $\omega_{1} \in \mathcal{H}_{0}$ which cannot be connected to $\omega_{0}$ by a smooth geodesic.

Concretely, one can take $X$ to be a torus $\mathbb{C}^{m} / \Gamma, \omega_{0}$ a translation invariant Kähler form, and $h$ induced by reflection $z \mapsto-z$ in $\mathbb{C}^{m}$.

According to Semmes, geodesics in $\mathcal{H}$ (and therefore in $\mathcal{H}_{0}$ ) are related to a Monge-Ampère equation as follows, [S]. Let $S=\{s \in \mathbb{C}: 0<\operatorname{Im} s<1\}$, and $\omega$ the pull back of $\omega_{0}$ by the projection $\bar{S} \times X \rightarrow X$. With any smooth curve $[0,1] \ni t \mapsto v_{t} \in \mathcal{H}$ associate the smooth function $u(s, x)=v_{\operatorname{Im} s}(x),(s, x) \in \bar{S} \times X$. Set $m=\operatorname{dim} X$. Then $t \mapsto v_{t}$ is a geodesic if and only if $u$ satisfies

$$
(\omega+i \partial \bar{\partial} u)^{m+1}=0 .
$$

2000 Mathematics Subject Classification. 32Q15, 32W20.

${ }^{1}$ The first author is grateful to the NSF for partial support (DMS 0700281) and to the Université Pierre et Marie Curie, Paris, where some of this research was done. 
Since $\omega+i \partial \bar{\partial} u$, restricted to fibers $\{s\} \times X$, is positive, (1.1) is equivalent to $\operatorname{rk} \omega+i \partial \bar{\partial} u \equiv m$; and so a smooth geodesic connecting $0, v \in \mathcal{H}$ gives rise to an $\omega$-plurisubharmonic $u \in C^{\infty}(\bar{S} \times X)$ solving

$$
\begin{aligned}
\operatorname{rk} \omega+i \partial \bar{\partial} u & \equiv m, \\
u(s+\sigma, x) & =u(s, x) \quad \text { for } \sigma \in \mathbb{R}, \quad(s, x) \in \bar{S} \times X, \\
u(s, x) & = \begin{cases}0, & \text { if } \operatorname{Im} s=0 \\
v(x), & \text { if } \operatorname{Im} s=1 .\end{cases}
\end{aligned}
$$

Therefore Theorem 1.1 follows from the following more precise result:

Theorem 1.2. If $\left(X, \omega_{0}\right)$ and $h$ are as in Theorem 1.1, there is a $v \in \mathcal{H}$ for which (1.2) admits no real valued solution $u \in C^{3}(\bar{S} \times X)$. One can choose $v$ so that $h^{*} v=v$.

When $m=1$, the $v$ in Theorem 1.2 even form an open subset of the space of $h$-invariant functions in $\mathcal{H}$, but we do not know if this holds when $m>1$.

The idea that symmetries help in the analysis of solutions of Monge-Ampère equations is not new. The first examples of irregularity of certain boundary value problems in $\mathbb{C}^{m}$ were constructed by Bedford and Fornaess using symmetries, see $[\mathrm{BF}]$. Our approach, based on the study of the so called Monge-Ampère foliation, is different from theirs. The symmetry will be used to identify a leaf of the foliation associated to a $C^{3}$ solution $u$ of (1.2). By analyzing the first order behavior of the foliation about this particular leaf we obtain a condition on the Hessian of $u$ at $\left(1, x_{0}\right)$, where $x_{0}$ is an isolated fixed point of $h$. The proof is concluded by finding a boundary value $v$ which is incompatible with this condition.

Studying solutions of the homogeneous Monge-Ampère equation through the associated foliation is not new, either. This approach first appeared in [Be1-2, L1$2]$, and still seems to be the only way to prove smoothness of the solution. More recently, in [D2] Donaldson used the foliation method in a variant of the boundary value problem (1.2) to prove, resp. disprove, regularity, depending on the boundary data.

Generalized solutions to (1.2) and to rather more general boundary value problems for the homogeneous Monge-Ampère equation (1.1) are known to exist, see [C], with complements in [Bt].

Most of this paper is devoted to the proof of Theorem 1.2. In section 4 we will discuss the implications of the theorem on the precise regularity of geodesics in $\mathcal{H}$.

Acknowledgement. We are grateful to B. Berndtsson and Z. Błocki for sparking our interest in the subject.

\section{The Monge-Ampère foliation.}

Let $Y$ be an $m+1$ dimensional complex manifold, $\omega$ a real $(1,1)$ form on it, of class $C^{1}, d \omega=0$. If $\mathrm{rk} \omega \equiv m$, the kernels of $\omega$ form an integrable subbundle of $T Y$, and so $Y$ is foliated by Riemann surfaces, whose tangent spaces are the kernels of $\omega$. The foliation is of class $C^{1}$. If $w$ is a locally defined potential of 
$\omega$, i.e. $i \partial \bar{\partial} w=\omega$, the section $\partial w$ of $T^{* 1,0} Y$ is holomorphic along any leaf of the foliation (see [BK, Theorem 2.4] for a more general statement). Applying this with $\omega$ replaced by $\omega+i \partial \bar{\partial} u$, we obtain

Proposition 2.1. Suppose $u \in C^{3}(\bar{S} \times X)$ is a real solution of (1.2). Then there is a foliation $\mathcal{F}_{u}$ on $\bar{S} \times X$, of class $C^{1}$, whose leaves are Riemann surfaces, tangent to $\operatorname{Ker}(\omega+i \partial \bar{\partial} u)$. If $w$ is a locally defined potential of $\omega$, then $\partial(w+u)$ is holomorphic along the leaves of $\mathcal{F}_{u}$.

$\mathcal{F}_{u}$ is called the Monge-Ampère foliation associated with $u$. We will also need

Proposition 2.2. Suppose $h: X \rightarrow X$ is a holomorphic map with a fixed point $x_{0}$. If $h^{2}=i d_{X}$, there are holomorphic local coordinates $z_{j}$ centered at $x_{0}$ in which $h$ is expressed as $\left(z_{j}\right) \mapsto\left( \pm z_{j}\right)$. All signs will be minus if $x_{0}$ is an isolated fixed point.

Proof. This is again not new. For any compact group of holomorphic transformations local coordinates centered at any fixed point can be found in which the transformations are linear, see e.g. [BM, p. 19]; and each linear transformation in this compact group is diagonalizable. In our case the eigenvalues of the linearization of $h$ must be \pm 1 , which means that in an eigenbasis it is indeed given by $\left(z_{j}\right) \mapsto\left( \pm z_{j}\right)$. Clearly, the fixed point is isolated only if all the signs are minus.

¿From now on we assume $X, \omega_{0}$, and $h$ are as in Theorem 1.2, and denote by $x_{0}$ an isolated fixed point of $h$. Let $\tilde{h}=\operatorname{id}_{\bar{S}} \times h: \bar{S} \times X \rightarrow \bar{S} \times X$. We also fix $v \in \mathcal{H}$ such that $h^{*} v=v$.

Proposition 2.3. If a real $u \in C^{3}(\bar{S} \times X)$ solves (1.2), then $\tilde{h}^{*} u=u$ and $\bar{S} \times\left\{x_{0}\right\}$ is one leaf of the Monge-Ampère foliation $\mathcal{F}_{u}$.

Proof. First observe that the solution to (1.2) is unique. Indeed, if $R=\{s \in$ $\mathbb{C}: 1<|s|<e\}$, any solution $u$ of $(1.2)$ defines a solution $U(s, x)=u(i \log s, x)$ of the homogeneous Monge-Ampère equation on $\bar{R} \times X$. The latter being a compact manifold with boundary, uniqueness for the boundary value problem on it is standard. One can argue as follows (see [D1, Corollary 7] for a slightly weaker statement). Denote by $\Omega$ the pullback of $\omega_{0}$ by the projection $\bar{R} \times X \rightarrow X$. The rank condition implies that the signature of $\Omega+i \partial \bar{\partial} U$ is constant in $\bar{R} \times X$; since $\Omega+i \partial \bar{\partial} U$ is semipositive at boundary points, it must be semipositive everywhere. Now suppose $U^{\prime}$ also solves $\operatorname{rk} \Omega+i \partial \bar{\partial} U^{\prime} \equiv m$, and $U=U^{\prime}$ on $\partial R \times X$. Upon adding a constant we can assume $U \geq 0$. With $\lambda>1$ and $U^{\prime \prime}=\lambda U^{\prime}-U$

$$
\left(\lambda \Omega+i \partial \bar{\partial} U+i \partial \bar{\partial} U^{\prime \prime}\right)^{m+1}=\lambda^{m+1}\left(\Omega+i \partial \bar{\partial} U^{\prime}\right)^{m+1}=0 .
$$

As $\lambda \Omega+i \partial \bar{\partial} U$ is positive on the fibers $\{s\} \times X$, by [D1, Lemma 6] $U^{\prime \prime}$ attains its minimum on $\partial R \times X$. (Donaldson speaks of maximum because he works with the operator $\bar{\partial} \partial$ rather than $\partial \bar{\partial}$.) But $U^{\prime \prime} \geq 0$ on $\partial R \times X$, hence everywhere, and so $U \leq \lambda U^{\prime}$; letting $\lambda \rightarrow 1$ gives $U \leq U^{\prime}$. Reversing the roles of $U$ and $U^{\prime}$ we see $U=U^{\prime}$.

Since $u$ and $\tilde{h}^{*} u$ solve the same boundary value problem, we conclude that they are indeed equal. It follows that $\operatorname{Ker}(\omega+i \partial \bar{\partial} u)$ is invariant under $\tilde{h}_{*}$, and so is 
$\left.\operatorname{Ker}(\omega+i \partial \bar{\partial} u)\right|_{\left(s, x_{0}\right)} \subset T_{\left(s, x_{0}\right)}(\bar{S} \times X)$, for any $s \in \bar{S}$. Proposition 2.2 shows that the only $\tilde{h}_{*}$-invariant complex lines in $T_{\left(s, x_{0}\right)}(\bar{S} \times X) \approx T_{s} \bar{S} \oplus T_{x_{0}} X$ are $T_{s} \bar{S} \oplus(0)$ and lines contained in $(0) \oplus T_{x_{0}} X$. Since $u \mid(\{0\} \times X)$ is strongly $\omega_{0}$-plurisubharmonic, $\left.\operatorname{Ker}(\omega+i \partial \bar{\partial} u)\right|_{\left(s, x_{0}\right)}$ must agree with $T_{s} \bar{S} \oplus(0)$ when $s=0$. By continuity, the same must hold for $s$ in a connected neighborhood $S^{\prime} \subset \bar{S}$ of 0 , and so $S^{\prime} \times\left\{x_{0}\right\}$ is contained in a leaf of $\mathcal{F}_{u}$. By analytic continuation, $\bar{S} \times\left\{x_{0}\right\}$ is itself a leaf, q.e.d.

In the rest of this section, for a real solution $u=\tilde{h}^{*} u \in C^{3}(\bar{S} \times X)$ of (1.2) we will study the first order behavior of $\mathcal{F}_{u}$ about the leaf $\bar{S} \times\left\{x_{0}\right\}$. We fix local coordinates $z_{j}, j=1, \ldots, m$, on a neighborhood $V \subset X$ of $x_{0}$, as in Proposition 2.2 ; we can choose them so that in addition $\left.\omega_{0}\right|_{x_{0}}=\left.i \sum d z_{j} \wedge d \bar{z}_{j}\right|_{x_{0}}$, and the local coordinates map $V$ on a convex set in $\mathbb{C}^{m}$. Using the coordinates we identify $V$ with its image in $\mathbb{C}^{m}$ and $x_{0}$ with $0 \in \mathbb{C}^{m}$. Then $\bar{S} \times V$ is identified with a subset of $\bar{S} \times \mathbb{C}^{m}$, and occasionally we shall write $z_{0}$ for the $s$ coordinate of a point $\left(s, z_{1}, \ldots, z_{m}\right) \in \bar{S} \times V$.

Exhaust $\bar{S}$ by compact subsets, say by rectangles

$$
S_{r}=\{s \in \bar{S}:|\operatorname{Re} s| \leq r\}, \quad r \in(0, \infty) .
$$

Given $r$, if $a \in V$ is sufficiently close to $x_{0}=0$, the leaf of $\mathcal{F}_{u} \mid S_{r} \times X$ passing through $(0, a)$ is the graph of a $C^{1}$ function $f_{a}: S_{r} \rightarrow V$, holomorphic on int $S_{r}$. For example, $f_{0} \equiv 0$. Let the components of $f_{a}$ be $f_{a j}: S_{r} \rightarrow \mathbb{C}, j=1, \ldots, m$, and let $f_{a 0}(s)=s$. Write

$$
\omega \mid \bar{S} \times V=i \sum_{j, k=0}^{m} \omega_{j k} d z_{j} \wedge d \bar{z}_{k}, \quad \text { with } \omega_{j k}=0 \text { if } j \text { or } k=0
$$

That the image of $\left(f_{a j}\right)_{j=0}^{m}$ is tangent to $\operatorname{Ker}(\omega+i \partial \bar{\partial} u)$ means

$$
\sum_{j=0}^{m}\left\{\omega_{j k}\left(s, f_{a}(s)\right)+u_{z_{j} \bar{z}_{k}}\left(s, f_{a}(s)\right)\right\} f_{a j}^{\prime}(s)=0, \quad k=0,1, \ldots, m .
$$

Further, if $\omega_{0} \mid V=i \partial \bar{\partial} w_{0}$ and $w(s, x)=w_{0}(x)$, then $\partial(w+u)$ is holomorphic along the (interior of the) leaves, i.e.,

$$
w_{z_{j}}\left(s, f_{a}(s)\right)+u_{z_{j}}\left(s, f_{a}(s)\right), \quad j=0,1, \ldots, m,
$$

depend holomorphically on $s \in \operatorname{int} S_{r}$.

Replace $a \in \mathbb{C}^{m}$ by $t a \in \mathbb{C}^{m}, t \in[0,1]$, and $f_{a}$ by $f_{t a}: S_{r(t)} \rightarrow V$, where $\lim _{t \rightarrow 0} r(t)=\infty$; then differentiate (2.1), (2.2) with respect to $t$ at $t=0$. Note that $\varphi_{j}=d f_{(t a) j} /\left.d t\right|_{t=0}$ are continuous functions on $\bar{S}$, holomorphic on $S$; and $\varphi_{0} \equiv 0$. The symmetry of $\omega, u$ implies that odd order derivatives of $\omega_{j k}$ and $u$, with respect to $z_{l}, \bar{z}_{l}, l \geq 1$, all vanish at $(s, 0)$. Hence differentiating (2.1) gives

$$
\sum_{l=1}^{m}\left\{u_{z_{l} \bar{z}_{k} s}(s, 0) \varphi_{l}(s)+u_{\bar{z}_{l} \bar{z}_{k} s}(s, 0) \overline{\varphi_{l}(s)}\right\}+\sum_{j=1}^{m}\left\{\delta_{j k}+u_{z_{j} \bar{z}_{k}}(s, 0)\right\} \varphi_{j}^{\prime}(s)=0
$$


$k=1, \ldots, m ;$ and $(2.2)$ gives that for $j=1, \ldots, m$

$$
\sum_{k=1}^{m}\left\{w_{0 z_{j} z_{k}}(0)+u_{z_{j} z_{k}}(s, 0)\right\} \varphi_{k}(s)+\sum_{k=1}^{m}\left\{w_{0 z_{j} \bar{z}_{k}}(0)+u_{z_{j} \bar{z}_{k}}(s, 0)\right\} \overline{\varphi_{k}(s)}
$$

are holomorphic. If $u_{z z}, u_{z \bar{z}}$, and $\varphi$ denote the $m \times m$ matrices $\left(u_{z_{j} z_{k}}\right),\left(u_{z_{j} \bar{z}_{k}}\right)$, and the column vector $\left(\varphi_{j}\right), j, k=1, \ldots, m$, then this latter says that

$$
u_{z z}(s, 0) \varphi(s)+\left(I+u_{z \bar{z}}(s, 0)\right) \overline{\varphi(s)}=\psi(s)
$$

is holomorphic.

Now $u(s, 0)$ depends only on $\operatorname{Im} s$. If we let

$$
P=I+u_{z \bar{z}}(i, 0)=I+v_{z \bar{z}}(0) \quad \text { and } \quad Q=u_{z z}(i, 0)=v_{z z}(0),
$$

(2.4) implies in light of (1.2)

$$
\psi(s)= \begin{cases}\overline{\varphi(s)}, & \text { if } \operatorname{Im} s=0 \\ P \overline{\varphi(s)}+Q \varphi(s), & \text { if } \operatorname{Im} s=1 .\end{cases}
$$

On the other hand, restricting (2.3) to real $s$ gives

$$
\varphi^{\prime}(s)=A \varphi(s)+B \overline{\varphi(s)}, \quad s \in \mathbb{R}
$$

where

$$
A=-u_{\bar{z} z s}(0,0)=-A^{*}, \quad B=-u_{\bar{z} \bar{z} s}(0,0)=B^{T} .
$$

That $A$ is skew adjoint follows from the translational invariance of the self adjoint matrix $u_{\bar{z} z}(s, 0)$, while the symmetry of $B$ is obvious. Finally we note

$$
\varphi(0)=a .
$$

The equations (2.6), (2.7), (2.9) describe the first order behavior of $\mathcal{F}_{u}$ about the leaf $\bar{S} \times\left\{x_{0}\right\}$. To summarize:

Proposition 2.4. For any $a \in \mathbb{C}^{m}$, the solution of the initial value problem (2.7), (2.9) can be extended to a continuous function $\varphi: \bar{S} \rightarrow \mathbb{C}^{m}$, holomorphic on $S$, and there is another continuous function $\psi: \bar{S} \rightarrow \mathbb{C}^{m}$, also holomorphic on $S$, that satisfies (2.6).

\section{The proof of Theorem 1.2 .}

We consider a real solution $u \in C^{3}(\bar{S} \times X)$ of the boundary value problem (1.2), with $v \in \mathcal{H}$ satisfying $h^{*} v=v$, and choose local coordinates $z_{j}$ centered at an isolated fixed point $x_{0}$ of $h$ as in section 2 . The main point of the proof is 
Lemma 3.1. Let $P, Q$ be defined by (2.5). Assume that $P$ and $Q$ have a common eigenbasis consisting of real vectors $\xi_{1}, \ldots, \xi_{m} \in \mathbb{R}^{m}$. If the eigenvalues of

$$
R=\left(I+P^{2}-Q \bar{Q}\right) P^{-1}
$$

are simple, then at least one of them is $\geq-2$.

One can even show that all the eigenvalues are $\geq-2$, but this is irrelevant to our purposes. We need an auxiliary result:

Proposition 3.2. Let $A=-A^{*}$ and $B=B^{T}$ be $m \times m$ complex matrices. If the block matrix

$$
M=\left(\frac{A}{B} \frac{B}{A}\right)
$$

has an eigenvector $\left(\begin{array}{l}x \\ y\end{array}\right)$ with eigenvalue $\lambda \in \mathbb{C}$, then either $\lambda^{2} \in \mathbb{R}$ or $|x|=|y|$.

Proof. One computes

$$
M^{2}=\left(\begin{array}{cc}
C & D \\
-D^{*} & E
\end{array}\right), \quad \text { with } \quad C^{*}=C, E^{*}=E .
$$

Denoting by $\langle$,$\rangle the Euclidean inner product on \mathbb{C}^{m}$, for $\xi, \xi^{\prime}, \eta, \eta^{\prime} \in \mathbb{C}^{m}$ we have

$$
\left\langle C \xi+D \eta, \xi^{\prime}\right\rangle-\left\langle-D^{*} \xi+E \eta, \eta^{\prime}\right\rangle=\left\langle\xi, C \xi^{\prime}+D \eta^{\prime}\right\rangle-\left\langle\eta,-D^{*} \xi^{\prime}+E \eta^{\prime}\right\rangle .
$$

Now substitute

$$
\left(\begin{array}{l}
\xi \\
\eta
\end{array}\right)=\left(\begin{array}{l}
\xi^{\prime} \\
\eta^{\prime}
\end{array}\right)=\left(\begin{array}{l}
x \\
y
\end{array}\right), \quad \text { so that }\left(\begin{array}{cc}
C & D \\
-D^{*} & E
\end{array}\right)\left(\begin{array}{l}
\xi \\
\eta
\end{array}\right)=\lambda^{2}\left(\begin{array}{l}
x \\
y
\end{array}\right)
$$

to obtain $\lambda^{2}\left(|x|^{2}-|y|^{2}\right)=\bar{\lambda}^{2}\left(|x|^{2}-|y|^{2}\right)$, and the claim follows.

Proof of Lemma 3.1. The assumptions imply that the $\xi_{k}$ are eigenvectors of $\bar{P}$ and $\bar{Q}$, with eigenvalues conjugate to the eigenvalues of $P$, resp. $Q$. As the eigenvalues of $P$ are real, it follows that $P=\bar{P}, Q$, and $\bar{Q}$ commute among themselves. Define $A, B$ by (2.8) and let $\left(\begin{array}{l}x \\ y\end{array}\right)$ be an eigenvector of the matrix (3.1), with eigenvalue $\lambda \in \mathbb{C}$. We apply Proposition 2.4. With $a=x+\bar{y}$ it is easy to write down the solution of the initial value problem $(2.7),(2.9)$ : it is

$$
\varphi(s)=x e^{\lambda s}+\bar{y} e^{\bar{\lambda} s}
$$

first for $s \in \mathbb{R}$, then by analytic continuation, for all $s \in \bar{S}$. Upon replacing the eigenvector by a multiple, we can arrange that $a=x+\bar{y} \neq 0$. ¿From the first equation in (2.6), $\psi(s)=\bar{x} e^{\bar{\lambda} s}+y e^{\lambda s}$. Substituting in the second, and noting that $\bar{s}=s-2 i$ when $\operatorname{Im} s=1$, we obtain

$$
P\left(\bar{x} e^{-2 i \bar{\lambda}} e^{\bar{\lambda} s}+y e^{-2 i \lambda} e^{\lambda s}\right)+Q\left(x e^{\lambda s}+\bar{y} e^{\bar{\lambda} s}\right)=\bar{x} e^{\bar{\lambda} s}+y e^{\lambda s} .
$$


If $\lambda \in \mathbb{R}$, we divide through by $e^{\lambda s}$ to get $P \bar{a}=e^{2 i \lambda}(I-Q) a$. Combining this equation with its complex conjugate yields

$$
\left\{(I-Q)(I-\bar{Q})-P^{2}\right\} a=0 .
$$

Let $P \xi_{j}=p_{j} \xi_{j}, Q \xi_{j}=q_{j} \xi_{j}$, with $p_{j}>0$. The eigenvalues of $(I-Q)(I-\bar{Q})-P^{2}$ are then $\left|1-q_{j}\right|^{2}-p_{j}^{2}$, and by (3.3) one of these numbers, say $\left|1-q_{1}\right|^{2}-p_{1}^{2}$, is zero. Hence $\left|q_{1}\right| \leq 1+p_{1}$, and the corresponding eigenvalue of $R,\left(1+p_{1}^{2}-\left|q_{1}\right|^{2}\right) / p_{1} \geq-2$, as claimed.

If $\lambda \notin \mathbb{R}$, then comparing the coefficients of $e^{\lambda s}$ and $e^{\bar{\lambda} s}$ in (3.2) yields

$$
Q x=\left(I-e^{-2 i \lambda} P\right) y, \quad Q \bar{y}=\left(I-e^{-2 i \bar{\lambda}} P\right) \bar{x} .
$$

Again, we combine these equations and their complex conjugates to get

$$
Q \bar{Q} x=\left(I-e^{-2 i \lambda} P\right)\left(I-e^{2 i \lambda} P\right) x, \quad Q \bar{Q} y=\left(I-e^{-2 i \lambda} P\right)\left(I-e^{2 i \lambda} P\right) y,
$$

or

$$
R x=\left(e^{-2 i \lambda}+e^{2 i \lambda}\right) x, \quad R y=\left(e^{-2 i \lambda}+e^{2 i \lambda}\right) y .
$$

So one eigenvalue of $R$ is $e^{-2 i \lambda}+e^{2 i \lambda}$. If $\lambda$ happens to be imaginary, then $e^{-2 i \lambda}+$ $e^{2 i \lambda} \geq 2$, as needed.

It remains to take care of the case when $\lambda$ is neither real nor imaginary. But in fact this case cannot occur. Indeed, Proposition 3.2 would imply $|x|=|y|$. Now $\xi_{1}, \ldots, \xi_{m}$ are eigenvectors of $R$, with different eigenvalues; in particular, $e^{-2 i \lambda}+e^{2 i \lambda}$ is a simple eigenvalue. Therefore by (3.4) $x$ and $y$ are proportional to each other, and to a real vector $\xi_{j}$. At the price of passing to a multiple, we can assume $x \in \mathbb{R}^{m}$, and then $y=\alpha x,|\alpha|=1$. We have

$$
\left(\begin{array}{cc}
\frac{A}{B} & \frac{B}{A}
\end{array}\right)\left(\begin{array}{c}
x \\
\alpha x
\end{array}\right)=\lambda\left(\begin{array}{c}
x \\
\alpha x
\end{array}\right),
$$

whence $A x+\alpha B x=\lambda x$ and $B x+\bar{\alpha} A x=\bar{\alpha} \bar{\lambda} x$. Multiplying the latter by $\alpha$ and subtracting from the former we obtain $0=(\lambda-\bar{\lambda}) x$, or $\lambda \in \mathbb{R}$, a contradiction. With this the proof of the lemma is complete.

It is easy to construct self adjoint, resp. symmetric, matrices $P>0$ and $Q$ that have a common real eigenbasis, but contrary to the claim of Lemma 3.1, the eigenvalues of $\left(I+P^{2}-Q \bar{Q}\right) P^{-1}$ are simple and $<-2$. For example one can take $P=I$ and $Q$ diagonal, with real eigenvalues $q_{1}>\ldots>q_{m}>2$. Theorem 1.2 would then follow from Lemma 3.1, if we could produce a strongly $\omega_{0}$-plurisubharmonic $v \in C^{\infty}(X)$ such that $h^{*} v=v$, and in the local coordinates constructed, $v_{z \bar{z}}\left(x_{0}\right)=$ $P-I, v_{z z}\left(x_{0}\right)=Q$. This can indeed be done:

Lemma 3.3. Let $\left(X, \omega_{0}\right)$ be a Kähler manifold with almost complex structure tensor $J: T X \rightarrow T X$, let $h: X \rightarrow X$ be a holomorphic isometry that fixes $x_{0} \in X$, and 
assume $h^{2}=i d_{X}$. Let $q$ be a real quadratic form on $T_{x_{0}} X$, invariant under $h_{*}$. If its Hermitian part

$$
q^{1,1}(\xi)=(q(\xi)+q(J \xi)) / 2, \quad \xi \in T_{x_{0}} X
$$

satisfies $q^{1,1}(\xi)+\omega_{0}(\xi, J \xi)>0$ for nonzero $\xi \in T_{x_{0}} X$, then there is a strongly $\omega_{0}$-plurisubharmonic $v \in C^{\infty}(X)$ such that $h^{*} v=v$, dv $\left(x_{0}\right)=0$, whose Hessian at $x_{0}$ is $q$.

The lemma is essentially the same as [D2, Lemma 8], except for the extra ingredient $h$. We also had to impose the condition on $q^{1,1}$. The proof to follow is a slight variation on Donaldson's proof.

Proof. We choose local coordinates $z_{j}$ centered at $x_{0}$ in which $h$ is expressed as $\left(z_{j}\right) \mapsto\left( \pm z_{j}\right)$. It is clear that in some neighborhood $U$ of $x_{0}$ there is a $w \in C^{\infty}(U)$ which has all the properties of $v$, except it is not defined on all of $X$. Indeed, if

$$
q=\sum_{j, k} a_{j k} d z_{j} d \bar{z}_{k}+\operatorname{Re} \sum_{j, k} b_{j k} d z_{j} d z_{k} \mid T_{x_{0}} X
$$

then $w=\left(\sum a_{j k} z_{j} \bar{z}_{k}+\operatorname{Re} \sum b_{j k} z_{j} z_{k}\right) / 2$ will do. By shrinking $U$ and by scaling the coordinates we can arrange that $i \partial \bar{\partial} w>(\delta-1) \omega_{0}$ on $U$, with some $\delta>0$, and that the coordinates map $U$ on a neighborhood of $\left\{z \in \mathbb{C}^{n}: \sum\left|z_{j}\right|^{2} \leq 1\right\}$.

Next choose a smooth function $\varphi:[-\infty, \infty) \rightarrow[0,1]$ such that

$$
\varphi(t)= \begin{cases}0, & \text { if } t>0 \\ 1, & \text { if } t<-1\end{cases}
$$

Then $\psi(t)=\varphi(\varepsilon \log t), \varepsilon>0$, defines a smooth function on $[0, \infty)$, supported on $[0,1]$, such that

$$
\psi(t)=1 \quad \text { if } t<e^{-1 / \varepsilon}, \quad \text { and } \quad t\left|\psi^{\prime}(t)\right|, t^{2}\left|\psi^{\prime \prime}(t)\right|<C \varepsilon
$$

with some $C$ independent of $\varepsilon$. Thus the smooth function

$$
v= \begin{cases}\psi\left(|z|^{2}\right) w & \text { on } U \\ 0 & \text { on } X \backslash U\end{cases}
$$

satisfies $d v\left(x_{0}\right)=0, h^{*} v=v$, and its Hessian at $x_{0}$ is $q$. Further, on $U$

$$
\omega_{0}+i \partial \bar{\partial} v>\omega_{0}+i \psi\left(|z|^{2}\right) \partial \bar{\partial} w-C^{\prime} \varepsilon \omega_{0}>\left(\delta-C^{\prime} \varepsilon\right) \omega_{0}
$$

with $C^{\prime}$ independent of $\varepsilon$. If $\varepsilon$ is small enough, $v$ therefore has all the required properties.

\section{The smoothness of geodesics.}

Even without delving into technicalities of infinite dimensional analysis, it is rather clear that if $X, \omega_{0}$, and $v \in \mathcal{H}$ are as in Theorem 1.2 , by this theorem there is no $C^{3}$ geodesic connecting 0 and $v$ in $\mathcal{H}$. It emerged from a conversation 
with Berndtsson that there is not even a $C^{2}$ geodesic, and in fact not even a geodesic of Sobolev class $W^{1,2}$ (this space is the largest in which the geodesic equation can be considered); but to prove this stronger result, we will have to delve into technicalities. Modulo technicalities though, the proof is just standard boot strapping for ordinary differential equations.

One technicality involves replacing $\mathcal{H}$ by Banach spaces. From now on $C^{k}(X)$ will denote the real Banach space of real functions of class $C^{k}$ on $X$. If $k=2,3, \ldots$ and $l=0,1, \ldots$, let

$$
\mathcal{H}^{k}=\left\{v \in C^{k}(X): \omega_{0}+i \partial \bar{\partial} v>0\right\}, \quad \mathcal{F}^{l}=\left\{\Omega \in C_{1,1}^{l}(X): \Omega>0\right\},
$$

open subsets of $C^{k}(X)$, resp. of the Banach space $C_{1,1}^{l}(X)$ of real $(1,1)$-forms of class $C^{l}$. We also write $C_{1,0}^{l}(X)$ for the Banach space of $(1,0)$ forms of class $C^{l}$. Another technicality is the notion of vector valued Sobolev spaces. Let $B$ be a Banach space and $J=[0,1]$. The notions of measurability, integrability, and $L^{p_{-}}$ integrability of functions $J \rightarrow B$ are rather obvious, and were codified in [Bo]. The weak derivative of $\beta \in L_{\text {loc }}^{1}(\operatorname{int} J ; B)$ is a function $\gamma \in L_{\text {loc }}^{1}(\operatorname{int} J ; B)$, denoted $\dot{\beta}$, such that $\int_{J} \varphi \gamma=-\int_{J} \dot{\varphi} \beta$ for all $\varphi \in C^{\infty}(J ; \mathbb{R}), \operatorname{supp} \varphi \subset(0,1)$. The only Sobolev space of $B$ valued functions of interest to us is

$$
W^{1,2}(J ; B)=\left\{\beta \in C(J ; B): \beta \text { has a weak derivative in } L^{2}(J ; B)\right\} .
$$

Mabuchi's Riemannian metric on $\mathcal{H}$ extends to a Riemannian metric on $\mathcal{H}^{2}$. The equation for geodesics $\beta: J \rightarrow \mathcal{H}$ involves a function

$$
F: \mathcal{F}^{0} \times C_{1,0}^{0}(X) \rightarrow C(X), \quad F(\Omega, f)=\langle f, f\rangle_{\Omega},
$$

the pointwise squared length of $f$, measured in the Hermitian metric determined by $\Omega$. By $[\mathrm{M},(3.1)]$ or $[\mathrm{S},(1.5)]$ the geodesic equation is

$$
\ddot{\beta}(t)=F\left(\omega_{0}+i \partial \bar{\partial} \beta(t), \partial \dot{\beta}(t)\right),
$$

and makes sense for $\beta: J \rightarrow \mathcal{H}^{2}$.

Proposition 4.1. For $l=0,1, \ldots, F$ defines a $C^{\infty}$ map $\mathcal{F}^{l} \times C_{1,0}^{l}(X) \rightarrow C^{l}(X)$. Furthermore, given a compact $K \subset \mathcal{F}^{l}$, there is a constant $c$ such that

$$
\|F(\Omega, f)\|_{C^{l}(X)} \leq c\|f\|_{C_{1,0}^{l}(X)}^{2}, \quad \text { for } \Omega \in K, f \in C_{1,0}^{l}(X) .
$$

Proof. It suffices to prove when the second argument $f$ in $F(\Omega, f)$ is supported in a coordinate neighborhood $N \subset X$. If in local coordinates

$$
\begin{gathered}
\Omega=i \sum \Omega_{j k} d z_{j} \wedge d \bar{z}_{k} \text { and } f=\sum f_{j} d z_{j}, \quad \text { then } \\
F(\Omega, f)=\sum \Omega^{j k} f_{j} \bar{f}_{k} \quad \text { in } N,
\end{gathered}
$$

and 0 elsewhere, with $\left(\Omega^{j k}\right)$ denoting the inverse of the matrix $\left(\Omega_{j k}\right)$. ¿From this formula both claims of the Proposition follow.

By Proposition 4.1, $F\left(\omega_{0}+i \partial \bar{\partial} \beta, \partial \dot{\beta}\right) \in L^{1}\left(J ; C^{k-2}(X)\right)$ if $\beta \in W^{1,2}\left(J ; \mathcal{H}^{k}\right)$. Hence the geodesic equation (4.2) can be considered for $\beta \in W^{1,2}\left(J ; \mathcal{H}^{k}\right)$ in the weak sense: we require that the weak derivative of $\dot{\beta} \in L^{2}\left(J ; C^{k}(X)\right) \subset L^{2}\left(J ; C^{k-2}(X)\right)$ should be $F\left(\omega_{0}+i \partial \bar{\partial} \beta, \partial \dot{\beta}\right)$. 
Theorem 4.2. If $X, \omega_{0}$, and $v$ are as in Theorem 1.2, and $k \geq 4$, then there is no $\beta \in W^{1,2}\left(J ; \mathcal{H}^{k}\right)$ that would satisfy $\beta(0)=0, \beta(1)=v$, and the geodesic equation (4.2) in the weak sense.

Proof. Of course, it suffices to prove when $k=4$. We will show that for any weak solution $\beta \in W^{1,2}\left(J ; \mathcal{H}^{4}\right)$ of $(4.2)$ the function

$$
w(t, x)=\beta(t)(x), \quad(t, x) \in J \times X
$$

is in $C^{3}(J \times X)$. In general, $w \in C^{l}(J \times X)$ if (and only if, but we will not need the reverse implication) $\beta \in C^{l-j}\left(J ; C^{j}(X)\right)$ for $j=0,1, \ldots l$. Indeed, when $l=0$, this follows directly from the definition of continuity. The case $l>0$ can be reduced to the case $l=0$ by observing that $w \in C^{l}(J \times X)$ if $\xi_{1} \ldots \xi_{l} w \in C(J \times X)$ for smooth vector fields $\xi_{i}$, each tangential either to the fibers $\{t\} \times X$ or to the fibers $J \times\{x\}$.

By the definition of $W^{1,2}\left(J ; \mathcal{H}^{4}\right)$,

$$
\beta \in C\left(J ; C^{3}(X)\right)
$$

Further, by Proposition 4.1 the map $t \mapsto F\left(\omega_{0}+i \partial \bar{\partial} \beta(t), \partial \dot{\beta}(t)\right) \in C^{2}(X)$ is integrable. Hence by (4.2) $t \mapsto \dot{\beta}(t) \in C^{2}(X)$ is continuous, or rather has a representative, continuous on $J$ (namely the one obtained by integrating $\ddot{\beta}$ ). In other words,

$$
\beta \in C^{1}\left(J ; C^{2}(X)\right)
$$

Feeding this and (4.3) back into (4.2), by Proposition 4.1 we obtain $\ddot{\beta}=F\left(\omega_{0}+\right.$ $i \partial \bar{\partial} \beta, \partial \dot{\beta}) \in C\left(J ; C^{1}(X)\right)$, or

$$
\beta \in C^{2}\left(J ; C^{1}(X)\right)
$$

Finally, feeding this and (4.4) back into into (4.2) once more gives $\ddot{\beta} \in C^{1}(J ; C(X))$, whence $\beta \in C^{3}(J ; C(X))$. This latter, together with (4.3), (4.4), and (4.5), implies $w \in C^{3}(J \times X)$, and therefore $u(s, x)=w(\operatorname{Im} s, x),(s, x) \in \bar{S} \times X$, is in $C^{3}(\bar{S} \times X)$. However, (4.3-5) also imply that the two sides of (4.2), viewed as elements of $C(X)$, agree for every $t \in J$, not just weakly. By the computations in [S] or [D1], this means that $\mathrm{rk} \omega_{0}+i \partial \bar{\partial} u \equiv m$, and of course the other equations in (1.2) are also satisfied. But if $v \in \mathcal{H}$ is suitably chosen, by Theorem 1.2 the solution of (1.2) cannot be in $C^{3}(\bar{S} \times X)$, and consequently $\beta$ cannot be in $W^{1,2}\left(J, \mathcal{H}^{4}\right)$.

\section{REFERENCES}

[Be1] E. Bedford, Extremal plurisubharmonic functions for certain domains in $\mathbb{C}^{2}$, Indiana U. Math. J. 28 (1979), 613-626.

[Be2] _ Stability of envelopes of holomorphy and the degenerate Monge-Ampère equation, Math. Ann. 259 (1982), 1-28.

[BF] E. Bedford, J. Fornaess, Counterexamples to regularity for the complex Monge-Ampère equation, Invent. Math. 50 (1979), 129-134. 
[BK] E. Bedford, M. Kalka, Foliations and complex Monge-Ampère equations, Comm. Pure Appl. Math. 30 (1977), 543-571.

[Bt] Z. Błocki, On geodesics in the space of Kähler metrics, Proc. Conf. in Geometry dedicated to Shing-Tung Yau (to appear).

[Bo] S. Bochner, Integration von Funktionen deren Werte die Elemente eines Vektorräumes sind, Fund. Math. 20 (1933), 262-276.

[BM] S. Bochner, W.T. Martin, Several complex variables, Princeton University Press, Princeton, 1948.

[C] X.X. Chen, The space of Kähler metrics, J. Diff. Geom. 56 (2000), 189-234.

[D1] S.K. Donaldson, Symmetric spaces, Kähler geometry and Hamiltonian dynamics, Amer. Math. Soc. Transl. Ser. 2, vol. 196, Amer. Math. Soc., Providence RI, 1999, pp. 13-33.

[D2] - Holomorphic discs and the complex Monge-Ampère equation, J. Sympl. Geom. 1 (2002), 171-196.

[L1] L. Lempert, La métrique de Kobayashi et la représentation des domaines sur la boule, Bull. Soc. Math. France 109 (1981), 427-474.

[L2] S Solving the degenerate complex Monge-Ampère equation with one concentrated singularity, Math. Ann. 263 (1983), 515-532.

[M] T. Mabuchi, Some symplectic geometry on compact Kähler manifolds I, Osaka J. Math. 24 (1987), 227-252.

[S] S. Semmes, Complex Monge-Ampère and symplectic manifolds, Amer. J. Math. 114 (1992), 495-550.

Department of Mathematics, Purdue University, West Lafayette, IN 47907, USA 